\title{
A NEW MOSS CHECKLIST OF NEGARA BRUNEI DARUSSALAM
}

\author{
Benito C. TAN \& Haji Mohamed
}

\begin{abstract}
A new moss checklist with updated nomenclature is given for the small country of Brunei Darussalam located in the northern part of Borneo. A total of 103 species in 50 genera are now collected and reported. The country's moss flora is still very much undercollected, judging from our present results: ( $i$ ) the absence of cosmopolitan and common paleotropical species such as Bryum apiculatum Schwägr., Callicostella papillata (Mont.) Mitt., Funaria hygrometrica Hedw., Isopterygium minutirameum (Müll. Hal.) A. Jaegr., Octoblepharum albidum Hedw. and Philonotis hastata (Duby) Wijk \& Margad.; (ii) the absence of widespread families such as Pottiaceae and Ditrichaceae; and (iii) the under-representation of speciose genera such as Ectropothecium, Macromitrium, Thuidium and Trichosteleum, with only one species collected. The incompleteness of our knowledge of the moss flora makes it impossible to assess the country's endangered moss species.
\end{abstract}

Key words: Borneo, Brunei Darussalam, Kuala Belalong, Mosses

Benito C. Tan, Department of Biological Science, National University of Singapore, Singapore 119267; also the University and Jepson Herbaria, University of California at Berkeley, Berkeley, CA 94720, USA.

Haji Mohamed, Dean's Office, Faculty of Science, University of Brunei Darussalam, Gadong, Brunei Darussalam BE1410

\section{INTRODUCTION}

The island of Borneo is a paradise of moss diversity. Recently the moss flora of the entire island was estimated to have 721 species in a checklist prepared by Suleiman et al. (2006), with the largest component, consisting of 582 species, reported from Sabah State, Malaysia. Yet up to 2009 only 40 species were recorded from Brunei Darussalam, a country located in the central part of the northern half of the island.

\section{GEOGRAPHICAL LOCATION AND VEGETATION OF BRUNEI DARUSSALAM}

The nation of Brunei (Negara Brunei Darussalam) is located on the north coast of the island of Borneo. Its northern coastline faces the South China Sea, and the other three sides of its national boundary are surrounded by Sarawak State, Malaysia. Further to the east of the country lies Sabah State, Malaysia, and in the distant south is the Kalimantan part of Indonesia.

The small human population of Brunei, reported at 401,000 in 2011 (internet information), inhabits a nation with a rich income derived mainly from the oil industry. The country boasts a large expanse of pristine rain forest left relatively untapped and not commercially exploited.

The country has a total area of 5770 square kilometers (Anonymous 2007). The climate type is warm and humid tropical equatorial, with $2800 \mathrm{~mm}$ average annual rainfall. Today about $60 \%$ of Brunei is still covered with lowland mixed dipterocarp rainforests. Some montane rain forests are scattered along its southern border with Sarawak State. Other types of vegetation found in Brunei include mangrove, peat swamp and heath forest (kerangas).

There are $c a 6000$ species of seed plants known from Brunei (Coode et al. 1996; Wong 1997), 99 of which are classified as threatened. The available estimate of the country's fern flora gives 450 species (see 'Brunei's fascinating ferns', Borneo Bulletin, June 19, 2000). Information about the local moss flora is meager in comparison. 
REVIEW OF BRYOLOGICAL HISTORY OF BRUNEI MOSS FLORA

Tan (2000) presented a short history of floristic study of the country's mosses, and prepared and issued the first set of Bruneian moss exsiccata, with 25 numbers belonging to 25 species in 22 genera. Of the 25 moss species represented in the set, 22 were then new records for the country. These newly documented species included several taxa widespread in the Malesian region: Arthrocormus schimperi, Fissidens ceylonensis, F. crassinervis, Leucobryum javense, Leucophanes glaucum, Microdus miquelianus (= Leptotrichella miqueliana), Meiothecium microcarpum and Vesicularia miquelii. This only shows the lamentable state of moss flora collection activity in Brunei.

Fortunately, with the implementation of the new transnational biodiversity project entitled 'Heart of Borneo', undertaken jointly by Malaysia, Indonesia and Brunei Darussalam, the diverse moss flora of Brunei has gained attention of late. The less explored eastern and southeastern parts of the country have been surveyed and extensively collected in recent years. As a result, below we add 24 genera and 63 species of mosses new to the country's flora, increasing the number of indigenous moss taxa to 103 species in 50 genera.

\section{NEW COLLECTION LOCALITY INFORMATION}

The new moss species records reported below were made by Prof. Haji Mohamed and his students at the University of Brunei Darussalam from two localities:

1 - Mixed dipterocarp forest in the Kuala Belalong Field Study Centre, mostly on trees, at 100-200 m a.s.1., Temburong District, 5-10 January 2009. Collection numbers shown are preceded by the letter 'B';

2 - Submontane heath forests at $840-1100 \mathrm{~m}$ a.s.l. in Temburong District near the Brunei/Sarawak border, collected with Saabiqah Abd. Salam and Liaw Lin-Ji, 3-8 July 2012. Collection numbers shown are without a preceding letter.

We combine below the newly found species records and the already published moss species from the country into a new checklist with updated nomenclature. Only new collections are provided with collection number(s) and locality and habitat information. The new generic and species records in the moss checklist are asterisked.

With the latest additional collections, the moss flora can now be more clearly seen as an extension of the rich moss flora of Borneo. To date, the country has no endemic moss species within its national boundary. However, it has two of the Bornean endemic mosses: Sclerohypnum littorale (Hampe) B. C. Tan and Octoblepharum arthrocormoides N. Salazar Allen \& B. C. Tan. The Bruneian moss flora is far from being completely surveyed. This is made clear by the absence of a number of cosmopolitan and common paleotropical species such as Bryum apiculatum Schwägr., Callicostella papillata (Mont.) Mitt., Funaria hygrometrica Hedw., Isopterygium minutirameum (Müll Hal.) A. Jaegr., Octoblepharum albidum Hedw. and Philonotis hastata (Duby) Wijk \& Margad., the absence of widespread families of Pottiaceae and Ditrichaceae, and the under-representation of speciose genera with only one species, such as Ectropothecium, Macromitrium, Thuidium and Trichosteleum, reported from the country. The incompleteness of our knowledge of the moss flora makes it impossible at present to assess the number of endangered moss species in the country.

\section{Mosses OF BRUNEI}

Acanthorrhynchium M. Fleisch. (Sematophyllaceae)

Acanthorrhynchium papillatum (Harv.) M. Fleisch. (see Suleiman et al. 2006) - Kuala Belalong Field Study Centre, 100-200 m, on bamboo, Haji Mohamed B1024, B1121.

*Acroporium Mitt. (Sematophyllaceae)

"Acroporium adspersum (Hampe) Broth. - Kuala Belalong Field Study Centre, 100-200 m, on tree, Haji Mohamed B1072, B1161.

*Acroporium convolutum (Bosch \& Sande Lac.) M. Fleisch. - Temburong District near border of Brunei and Sarawak, 840-1100 m, on tree, Haji Mohamed et al. $68,104$. 
*Acroporium diminutum (Brid.) M. Fleisch. - Temburong District near border of Brunei and Sarawak, $860 \mathrm{~m}$, on branches, Haji Mohamed et al. 9 .

*Acroporium downii (Dixon) Broth. - Temburong District near border of Brunei and Sarawak, $860 \mathrm{~m}$, on tree, Haji Mohamed et al. 14, 85.

*Acroporium johannis-winkleri Broth. - Temburong District near border of Brunei and Sarawak, 840$1100 \mathrm{~m}$, on tree, Haji Mohamed et al. 4, 5, 20, 50,92 .

*Acroporium lamprophyllum Mitt. - Temburong District near border of Brunei and Sarawak, $860 \mathrm{~m}$, on tree and fallen twigs, Haji Mohamed et al. 90, 93.

${ }^{*}$ Acroporium rigens (Dixon) Dixon - Temburong District near border of Brunei and Sarawak, 840$1100 \mathrm{~m}$, on trees, Haji Mohamed et al. 10, 23, 67, $88,91,105$.

Acroporium rufum (Reinw. \& Hornsch.) M. Fleisch. - Temburong District near border of Brunei and Sarawak, 900 m, on Agathis tree, Haji Mohamed et al. 121.

${ }^{*}$ Acroporium secundum (Reinw. \& Hornsch.) M. Fleisch. - Temburong District near border of Brunei and Sarawak, 840 m, on log, Haji Mohamed et al. 24.

${ }^{*}$ Acroporium stramineum (Reinw. \& Hornsch.) M. Fleisch. - Temburong District near border of Brunei and Sarawak, $840 \mathrm{~m}$, on tree, Haji Mohamed et al. 125.

*Acroporium strepsiphyllum (Mont.) B. C. Tan - Temburong District near border of Brunei and Sarawak, 900 m., on tree, Haji Mohamed et al. 55.

*Aerobryopsis M. Fleisch. (Meteoriaceae)

*Aerobryopsis subleptostigmata Broth. \& Paris. Kuala Belalong Field Study Centre, 100-200 m, on branches, Haji Mohamed B1056.

Arthrocormus Dozy \& Molk. (Calymperaceae)

Arthrocormus schimperi (Dozy \& Molk.) Dozy \& Molk. (see Suleiman et al. 2006).

${ }^{*}$ Callicostella (Müll. Hal.) Mitt. (Pilotrichaceae)

*Callicostella prabaktiana (Müll. Hal.) Bosch \& Sande Lac. - Kuala Belalong Field Study Centre, 100$200 \mathrm{~m}$, on partially submerged logs in stream, colls. Haji Mohamed B1023, B1122, B1217.
Calymperes Sw. ex F. Weber (Calymperaceae)

"Calymperes fasciculatum Dozy \& Molk. - Kuala Belalong Field Study Centre, 100-200 m, on rocks along river, Haji Mohamed B1085, B1214.

${ }^{*}$ Calymperes lonchophyllum Schwägr. - Kuala Belalong Field Study Centre, 100-200 m, on base of tree, Haji Mohamed B1095, B1201, B1209, B1153, B1230.

Calymperes moluccense Schwägr. (see Suleiman et al. 2006).

Calymperes motleyi Mitt. (see Suleiman et al. 2006).

${ }^{*}$ Calymperes palisotti Schwägr. - Kuala Belalong Field Study Centre, 100-200 m, on base of tree, Haji Mohamed B1113, B1042.

${ }^{*}$ Calymperes porrectum Mitt. - Kuala Belalong Field Study Centre, 100-200 m, on boulder, Haji Mohamed B1014, B1041 (pro parte, on base of tree, mixed with Mniomalia semilimbata), B1214.

*Calymperes strictifolium (Mitt.) G. Roth - Kuala Belalong Field Study Centre, 100-200 m, on base of tree, coll. Haji Mohamed B1040.

Chaetomitrium Dozy \& Molk. (Symphyodontaceae)

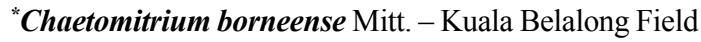
Study Centre, on twigs, Haji Mohamed B1203.

${ }^{*}$ Chaetmitrium orthorrhynchum (Dozy \& Molk.) Bosch \& Sande Lac. - Kuala Belalong Field Study Centre, 100-200 m, on twigs, Haji Mohamed B1021, B1074.

Chaetomitrium setosum Broth. ex Dix. (see Suleiman et al. 2006).

"Cladopodanthus Dozy \& Molk. (Leucobryaceae)

${ }^{*}$ Cladopodanthus speciosus (Dozy \& Molk.) M. Fleisch. - Temburong District near border of Brunei and Sarawak, $890 \mathrm{~m}$, on base of tree, coll. Haji Mohamed et al. 63.

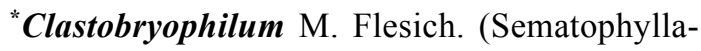
ceae)

"Clastobryophilum bogoricum (Bosch \& Sande Lac.) M. Fleisch. - Kuala Belalong Field Study Centre, 100-200 m, on fallen leaves and twigs, coll. Haji Mohamed B1181. 
${ }^{*}$ Clastobryum Dozy \& Molk. (Sematophyllaceae)

* Clastobryum cuculligerum (Sande Lac.) Tixier Kuala Belalong Field Study Centre, 100-200 m, coll. Haji Mohamed B1053 (on branch).

${ }^{*}$ Ctenidialphus M. Flesich. (Hypnaceae)

${ }^{*}$ Ctenidialphus plumularia (Müll. Hal.) M. Fleisch. Kuala Belalong Field Study Centre, 100-200 m, on tree, colls. Haji Mohamed B1087, B1094, B1106, B1011.

Dicranoloma (Renauld) Renauld (Dicranaceae)

Dicranoloma assimile (Hampe) Paris (see Suleiman et al. 2006).

*Dicranoloma blumii (Nees) Paris - Temburong District near border of Brunei and Sarawak, 840-1100 m, on tree near stream, coll. Haji Mohamed et al. 27.

*Dicranoloma reflexum (Müll. Hal.) Renauld - Temburong District near border of Brunei and Sarawak, $860 \mathrm{~m}$, on tree and twigs and exposed soil, Haji Mohamed et al. 129, 89.

\section{Diphyscium D. Mohr (Diphysciaceae)}

*Diphyscium mucronifolium Mitt. - Temburong District near border of Brunei and Sarawak, $840 \mathrm{~m}$, on rock near stream, Haji Mohamed et al. 87.

Diphyscium rupestre Dozy \& Molk. (see Suleiman et al. 2006).

*Distichophyllum Dozy \& Molk. (Daltoniaceae)

${ }^{*}$ Distichophyllum jungermannoides (Müll. Hal.) Bosch \& Sande Lac. - Kuala Belalong Field Study Centre, 100-200 m, on tree, Haji Mohamed B1081, B1105 (on tree in Earthwatch plot).

"Distichophyllum kinabaluense Nog. \& Z. Iwats. Temburong District near border of Brunei and Sarawak, about $200 \mathrm{~m}$, on wet soil, Haji Mohamed et al. $64,11,115$.

\section{Ectropothecium Mitt. (Hypnaceae)}

Ectropothecium dealbatum (Reinw. \& Hornsch.) Jaeg. (see Suleiman et al. 2006)

"Ectropothecium monumentorum (Duby) A. Jaeger Kuala Belalong Field Study Centre, 100-200 m, on tree, colls. Haji Mohamed B1072, B1034.

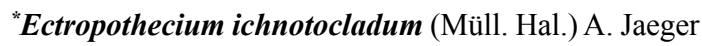

- Kuala Belalong Field Study Centre, 100-200 m, on tree, coll. Haji Mohamed B1189.

"Exostratum L. T. Ellis (Calymperaceae)

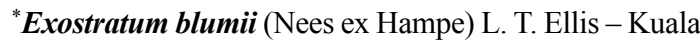
Belalong Field Study Centre, 100-200 m, on trees, Haji Mohamed B1067, B1123a, B1231.

Fissidens Hedw. (Fissidentaceae)

Fissidens ceylonensis Dozy \& Molk. (see Suleiman et al. 2006).

Fissidens crassinervis Sande Lac. (see Suleiman et al. 2006).

*Fissidens crispulus Brid. var. crispulus - Kuala Belalong Field Study Centre, 100-200 m, on tree, coll. Haji Mohamed B1027.

${ }^{*}$ Fissidens crispulus Brid. var. robinsonii (Broth.) Z. Iwats. \& Z.-H. Li - Temburong District near border of Brunei and Sarawak, $840 \mathrm{~m}$, on rock near stream, Haji Mohamed et al. 87.

${ }^{*}$ Fissidens hollianus Dozy \& Molk. - Kuala Belalong Field Study Centre, 100-200 m., on tree, Haji Mohamed B1220.

*Fissidens javanicus Dozy \& Molk. - Kuala Belalong Field Study Centre, 100-200 m, on bank of stream, Haji Mohamed B1029, B1158 (pro parte), B1047.

*Fissidens nobilis Griff. - Kuala Belalong Field Study Centre, 100-200 m, on bank of stream, Haji Mohamed B1158.

${ }^{*}$ Fissidens zollingeri Mont. - Kuala Belalong Field Study Centre, 100-200 m, on soil, Haji Mohamed B1190.

*Garovaglia Endl. (Pterobryaceae)

${ }^{*}$ Garovaglia compressa Mitt. - Kuala Belalong Field Study Centre, 100-200 m, on tree sapling, Haji Mohamed B1022.

"Himantocladium (Mitt.) M. Flesich. (Neckeraceae)

${ }^{*}$ Himantocladium plumula (Nees) M. Fleisch. - Kuala Belalong Field Study Centre, 100-200 m, on tree, Haji Mohamed B1124.

*Hypnodendron (Müll. Hal.) S. O. Lindberg ex Mitt. (Hypnodendraceae)

${ }^{*}$ Hypnodendron spininervium (Hook.) A. Jaeger \& Sauerb. - Kuala Belalong Field Study Centre, 100-200 m, 
on exposed root in moist area, Haji Mohamed B1231a.

"Hypnodendron dendroides (Brid.)Touw. - Temburong District near border of Brunei and Sarawak, $860 \mathrm{~m}$, on tree and wet ground, Haji Mohamed et al. 54.

Isocladiella Dixon (Sematophyllaceae)

Isocladiella surcularis (Dixon) B. C. Tan \& Mohamed (see Suleiman et al. 2006).

Leptotrichella (Müll. Hal.) Lindb. (Dicranaceae)

Leptotrichella miqueliana (Mont.) S. O. Lindberg ex Broth. [syn. Microdus miquelianus (Mont.) Besch.] (see Suleiman et al. 2006).

\section{Leucobryum Hampe (Leucobryaceae)}

Leucobryum aduncum (Bosch. \& Sande Lac.) M. Fleisch. var. scalare (Müll. Hal. ex M. Fleisch.) A. Eddy (see Suleiman et al. 2006) - Temburong District near border of Brunei and Sarawak, 840$1100 \mathrm{~m}$, on tree, Haji Mohamed et al. 78.

${ }^{*}$ Leucobryum candidum (Brid. ex P. Beauv.) Wilson - Temburong District near border of Brunei and Sarawak, Haji Mohamed et al. 77 (no habitat information); Kuala Belalong Field Study Centre, 100-200 m, on log, Haji Mohamed B1146.

${ }^{*}$ Leucobryum chlorophyllosum Müll. Hal. - Temburong District near border of Brunei and Sarawak, $860 \mathrm{~m}$, on tree, Haji Mohamed et al. 71 (in part), 132.

${ }^{*}$ Leucobryum javense (Brid.) Mitt. (see Suleiman et al. 2006) - Temburong District near border of Brunei and Sarawak, 860-900m, on humus, Haji Mohamed et al 2, 46, 102.

${ }^{*}$ Leucobryum sanctum (Brid.) Hampe - Kuala Belalong Field Study Centre, 100-200 m, on tree, Haji Mohamed B1059.

\section{Leucophanes Brid. (Calymperaceae)}

Leucophanes glaucum (Schwägr.) Mitt. (see Suleiman et al. 2006)

Leucophanes candidum (Schwägr.) Lindb. - Kuala Belalong Field Study Centre, 100-200 m, on log, Haji Mohamed B1130.

${ }^{*}$ Leucophanes octoblepharioides Brid. - Kuala Belalong Field Study Centre, 100-200 m, on tree, Haji Mohamed B1119.
"Macromitrium Brid. (Orthotrichacae)

*Macromitrium ochraceum (Dozy \& Molk.) Müll. Hal. - Temburong District near border of Brunei and Sarawak, on tree, Haji Mohamed et al. 42, 80, $100,114,124$.

\section{Mastopoma Cardot (Sematophyllaceae)}

Mastopoma brauniana (Sande Lac.) H. Akiyama [syn. Trismegistia brauniana (Lac.) M. Flesich.] (see Suleiman et al. 2006).

${ }^{*}$ Mastopoma uncinifolium (Broth.) Broth. - Temburong District near border of Brunei and Sarawak, $1070 \mathrm{~m}$., on tree, Haji Mohamed et al. 131.

Meiothecium Mitt. (Sematophyllaceae)

Meiothecium microcarpum (Hook.) Mitt. (see Suleiman et al. 2006).

"Meteorium (Bridel) Dozy \& Molkenboer (Meteoriaceae)

"Meteorium polytrichum Dozy \& Molk. [syn. M. miquelianum (Müll. Hal.) M. Fleisch.] - Temburong District near border of Brunei and Sarawak, $870 \mathrm{~m}$, on tree, Haji Mohamed et al. 114.

Mitthyridium H. Rob. (Calymperaceae)

*Mitthyridium crassum (Broth.) H. Rob. - Kuala Belalong Field Study Centre, $100-200 \mathrm{~m}$, on fallen trees, Haji Mohamed B1037, B1049, B1057.

${ }^{*}$ Mitthyridum fasciculatum (Hook. \& Grev.) H. Rob. Kuala Belalong Field Study Centre, 100-200 m, on branch and tree, Haji Mohamed B1104, B1147.

${ }^{*}$ Mitthyridium flavum (Müll. Hal.) H. Rob. - Kuala Belalong Field Study Centre, 100-200 m, on tree, Haji Mohamed B1001, B1063, B1067, B1099, B1113, B1200.

Mitthyridium jungquilianum (Mitt.) H. Rob. (see Suleiman et al. 2006).

${ }^{*}$ Mitthyridium undulatum (Dozy \& Molk.) H. Rob. Kuala Belalong Field Study Centre, 100-200 m, on trees and branches, Haji Mohamed B1078, B1108, B1104, B1208.

Mitthyridium wallisii (Muell. Hal.) H. Rob. (see Suleiman et al. 2006). 
Mniomalia Müll. Hal. (Phyllodrepaniaceae)

${ }^{*}$ Mniomalia semilimbata (Mitt.) Müll. Hal. (see Suleiman et al. 2006) - Kuala Belalong Field Study Centre, 100-200 m, on tree, Haji Mohamed B1009.

\section{Neckeropsis Reichardt (Neckeraceae)}

Neckeropsis beccariana (Hampe) A. Touw [syn. Nekkeropsis fleischeri (Dixon) A. Touw] (Suleiman et al. 2006)

${ }^{*}$ Neckeropsis gracilenta (Bosch \& Sande Lac.) M. Flesich. - Kuala Belalong Field Study Centre, 100-200 m, on bark of tree near river, Haji Mohamed B1123.

\section{Octoblepharum Hedw. (Octoblepharaceae)}

Octoblepharum arthrocormoides N. Salazar Allen \& B. C. Tan (see Salazar Allen \& Tan 2010). The type specimen is from the kerangas forest in the Temburong District of Brunei. The species is also found from a limestone area in Sarawak in North Borneo.

Oedicladium Mitt. (Myuriaceae)

Oedicladium pseudorufescens (Hampe) B. C. Tan $\&$ Mohamed [syn. Piloecium pseudorufescens (Hampe) Müll. Hal.] (see Suleiman et al. 2006).

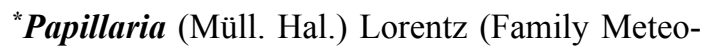
riaceae)

${ }^{*}$ Papillaria fuscescens (Hook.) A. Jaeger. - Temburong District near border of Brunei and Sarawak, $850 \mathrm{~m}$, on tree, Haji Mohamed et al 66.

*Papillidiopsis W. R. Buck \& B. C. Tan (Sematophyllaceae)

${ }^{*}$ Papillidiopsis malesiana W. R. Buck \& B. C. Tan Kuala Belalong Field Study Centre, 100-200 m, on tree and branches, Haji Mohamed B1058, B1038.

"Papillidiopsis bruchii (Dozy \& Molk.) W. R. Buck \& B. C. Tan - Kuala Belait, Bukit Sawat, UBD plot, on tree, 100 m, Noramaliyana Haji Manaf 66, 161.

Pinnatella M. Fleisch. (Neckeraceae)

Pinnatella anacamptolepis (Müll. Hal.) Broth. (see Suleiman et al. 2006)

${ }^{*}$ Pinnatella mucronata (Bosch \& Sande Lac.) M. Flesich. - Kuala Belalong Field Study Centre, 100-
$200 \mathrm{~m}$, on tree, Haji Mohamed B1012, B1084, $B 1112$.

*Pogonatum P. Beauv. (Polytrichaceae)

${ }^{*}$ Pogonatum piliferum (Dozy \& Molk.) A. Touw - Temburong District near border of Brunei and Sarawak, $850 \mathrm{~m}$, on dry stream bank, Haji Mohamed et al. 01, 47, 79; Kuala Belalong Field Study Centre, 100-200 m, on dry stream bank, Haji Mohamed B1103.

${ }^{*}$ Pogonatum proliferum (Griff.) Mitt. - Temburong District near border of Brunei and Sarawak, $850 \mathrm{~m}$, on soil, Haji Mohamed et al. 51, 103.

*Pyrrhobryum Mitt. (Rhizogoniaceae)

*Pyrrhobryum latifolium (Bosch \& Sande Lac.) Mitt. - Temburong District near border of Brunei and Sarawak, 829 m, on trunk, Haji Mohamed et al. 40, 123.

${ }^{*}$ Pyrrhobryum spiniforme (Hedw.) Mitt. - Temburong District near border of Brunei and Sarawak, $900 \mathrm{~m}$, on tree, Haji Mohamed et al. 41, 99.

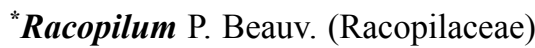

${ }^{*}$ Racopilum cuspidigerum (Schwägr.) Ĺngström - Kuala Belalong Field Study Centre, 100-200 m, on bark of tree, Haji Mohamed B1090.

"Radulina W. R. Buck \& B. C. Tan (Sematophyllaceae)

${ }^{*}$ Radulina borbonica (Bél.) W.R. Buck [syn. Radulina hamata (Dozy \& Molk.) W. R. Buck \& B. C. Tan] - Temburong District near border of Brunei and Sarawak, $840 \mathrm{~m}$, on rocks in river, Haji Mohamed et al. 52, 119; Kuala Belalong Field Study Centre, 100-200 m, on tree, Haji Mohamed B1052.

${ }^{*}$ Rhizogonium Brid. (Rhizogoniaceae)

${ }^{*}$ Rhizogonium graeffeanum (Müll. Hal.) A. Jaeger - Temburong District near border of Brunei and Sarawak, 840 m, on tree, Haji Mohamed et al. 45.

"Schistomitrium Dozy \& Molk. (Leucobryaceae)

${ }^{*}$ Schistomitrium apiculatum (Dozy \& Mok.) Dozy \& Molk. - Temburong District near border of Brunei and Sarawak, $860 \mathrm{~m}$, on tree, Haji Mohamed et al. 13, 71 (in part), 122. 


\section{Sclerohypnum Dixon (Hypnaceae)}

Sclerohypnum littorale (Hampe) B. C. Tan (see Suleiman et al. 2006)

\section{Sphagnum L. (Sphagnaceae)}

Sphagnum cuspidatum Hoffm. (see Suleiman et al. 2006)

Syrrhopodon Schwägr. (Calymperaceae)

Syrrhopodon albovaginatus Schwägr. (see Suleiman et al. 2006).

Syrrhopodon aristifolius Mitt. (see Suleiman et al. 2006).

Syrrhopodon ciliatus (Hook.) Schwägr. (see Suleiman et al. 2006).

Syrrhopodon confertus Sande Lac. (see Suleiman et al. 2006).

Syrrhopodon croceus Mitt. (see Suleiman et al. 2006).

${ }^{*}$ Syrrhopodon hispidissimus Dixon - Kuala Belalong Field Study Centre, 100-200 m, on tree Haji Mohamed B1066 (pro parte), B1210.

Syrrhopodon involutus Schwägr. (see Suleiman et al. 2006).

Syrrhopodon loreus (Lac.) Reese (see Suleiman et al. 2006).

Syrrhopodon muelleri (Dozy \& Molk.) Sande Lac. (see Suleiman et al. 2006).

Syrrhopodon spiculosus Hook. \& Grev. (see Suleiman et al. 2006).

${ }^{*}$ Syrrhopodon tristichus Nees ex Schwägr. - Kuala Belalong Field Study Centre, 100-200 m, on trees near river, Haji Mohamed B1013, B1050, B1051, B1192, $B 1071$ (mixed with $S$. loreus).

Taxithelium Spruce ex Mitt. (Sematophyllaceae)

${ }^{*}$ Taxithelium instratum (Brid.) Broth. - Kuala Belalong Field Study Centre, 100-200 m, on exposed root stump, Haji Mohamed B1190, B1198, B1231b.

${ }^{*}$ Taxithelium isocladum (Bosch \& Sande Lac.) Renauld \& Cardot - Temburong District near border of Brunei and Sarawak, 100-200 m, on roots, Haji Mohamed et al. 1015, 1018; Kuala Belalong Field Study Centre, 100-200 m, on base of tree, Haji Mohamed B1066 (pro parte, mixed with Syrrhopodon hispidissimus), B1115.

Taxithelium merrillii Broth. (see Suleiman et al. 2006).
*Thuidium Bruch \& Schimp. (Thuidiaceae)

${ }^{*}$ Thuidium kuripanum (Dozy \& Molk.) R. Watan. Kuala Belalong Field Study Centre, 100-200 m, on tree, Haji Mohamed B1079.

Trichosteleum Mitt. (Sematophyllaceae)

Trichosteleum procerum Dixon var. laevifolium Dixon (see Suleiman et al. 2006).

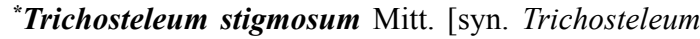
singapurense M. Fleisch.] - Kuala Belalong Field Study Centre, 100-200 m, on tree, Haji Mohamed B1006a, B1116, B1117.

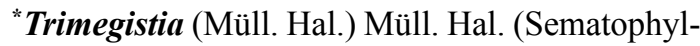
laceae)

${ }^{*}$ Trimegistia brachyphylla M. Fleisch. - Temburong District near border of Brunei and Sarawak, $860 \mathrm{~m}$, on tree, Haji Mohamed et al. 51.

Trismegistia lancifolia (Harv.) Broth. var. lancifolia (see Akiyama 2010).

Trismegistia lancifolia (Harv.) Broth. var. valetonii (M. Fleisch. ex Dixon) H. Akiyama (see Akiyama 2010).

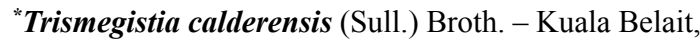
Bukit Sawat, UBD plot, on tree, 100 m, Noramaliyana Haji Manaf 199.

Vesicularia (Müll. Hal.) Müll. Hal. (Hypnaceae)

${ }^{*}$ Vesicularia dubyana (Müll. Hal.) Broth. - Kuala Belalong Field Study Centre, 100-200 m, on soil, Haji Mohamed B1068.

Vesicularia miquelii (Lac.) Fleisch. (see Suleiman et al. 2006).

ACKNOWLEDGMENTS. The first author acknowledges the funding provided by the University of Brunei Darussalam for his short visit in October of 2012 to study the new moss collections made by Professor Haji Mohamed and his students which form the main part of the new information reported here.

\section{REFERENCES}

AKIYAmA, H. 2010. Taxonomic revision of the genus Trismegistia (Pylaisiadelphaceae, Musci). Humans and Nature 21: 1-77.

ANONYMOUS 2007. Brief on National Forest Inventory: Brunei Darussalam. MAR-SFM Working Paper 4: 1-19. 
Coode M. J. E., Dransfield J., Forman L. L., KirkuP D. W. \& SAID IDRIS M. 1996. A checklist of the flowering plants and gymnosperms of Brunei Darussalam. Ministry of Industry and Primary Resources, Brunei Darussalam.

SAlazAR Allen N. \& TAN B. C. 2010. Octoblepharum arthrocormoides (Calymperaceae) N. Salazar Allen \& B. C. Tan, sp. nov., a new species from tropical Asia. Botany 88(4): 439-442.

Suleiman M., AkiYama H. \& TAN B. C. 2006. A revised catalogue of mosses reported from Borneo. J. Hattori Bot. Lab. 99: 107-183.
TAN B. C. 2000. The first moss exsiccata of Brunei Darussalam issued by the Raffles Museum for Biodiversity Research, National University of Singapore. Raffles Bulletin of Zoology 48: 338.

WONG K. M. 1997. In Brunei forests: an introduction to the plant life of Brunei Darussalam. Natural History Publications in association with Forestry Department, Ministry of Industry and Primary Resources, Brunei Darussalam.

Received 12 May 2013 